\title{
Soziale Netzwerke und die Gesundheit von Alleinerziehenden
}

\author{
Sylvia Keim-Klärner
}

\section{Überblick}

- Alleinerziehende verfügen über eine schlechtere psychische und physische Gesundheit als verheiratete Eltern.

- Die Relevanz sozialer Beziehungen und sozialer Unterstützung für das Wohlbefinden und die Gesundheit Alleinerziehender wurde vielfach belegt.

- Auch die Relevanz sozialer Netzwerke und ihrer Charakteristika konnte in einigen wenigen Studien aufgezeigt werden. Insgesamt sind spezifisch netzwerkanalytische Studien zur Gesundheit Alleinerziehender selten.

- Netzwerkstudien zur Gesundheit Alleinerziehender sind aber besonders relevant, weil

- wir wenig über den Zusammenhang zwischen spezifischen Netzwerkstrukturen und der Gesundheit Alleinerziehender wissen,

- sie über das Konzept der sozialen Unterstützung hinausgehen und auch andere Wirkmechanismen einbeziehen,

- sie auch negative und ambivalente Beziehungsinhalte einbeziehen und damit der Komplexität sozialer Beziehungsgeflechte in besonderem

S. Keim-Klärner $(\bowtie)$

Braunschweig, Deutschland

E-Mail: sylvia.keim-klaerner@thuenen.de

(C) Der/Die Herausgeber und der/die Autor(en) 2020

A. Klärner et al. (Hrsg.), Soziale Netzwerke und gesundheitliche Ungleichheiten, https://doi.org/10.1007/978-3-658-21659-7_14 
Maße gerecht werden und dazu beitragen, das Zusammenspiel von unterstützenden und konfliktbehafteten Beziehungen näher zu untersuchen,

- wir noch wenig darüber wissen, unter welchen Umständen und inwieweit welche Arten von sozialen Netzwerken in der Lage sind, soziale Ungleichheiten zu kompensieren, sodass diese nicht gesundheitsrelevant werden.

\section{$1 \quad$ Einleitung}

Einelternfamilien, das heißt Mütter oder Väter, die zusammen mit ihren Kindern, aber ohne einen Partner oder eine Partnerin im selben Haushalt leben, sind keine Seltenheit mehr. Die Lebensform „Alleinerziehend“ hat sich in Deutschland im Verlauf der letzten Jahrzehnte etabliert. Mittlerweile gibt es 1,6 Mio. Alleinerziehende mit Kindern unter 18 Jahren und rund jede fünfte Familie mit minderjährigen Kindern ist eine Einelternfamilie (Statistisches Bundesamt 2018, S. 55, 59). Alleinerziehende sind zumeist Frauen, nur etwa $12 \%$ der Alleinerziehenden sind Männer (Statistisches Bundesamt 2018, S. 55).

Auch wenn diese Lebensform häufiger auftritt und nicht mehr so stark gesellschaftlich stigmatisiert wird wie in früheren Zeiten, sind mit ihr doch ganz spezifische Risiken verbunden: So sind 2014 rund ein Drittel aller Alleinerziehenden von Armut bedroht, während dies nur für etwa ein Zehntel der Paarhaushalte mit zwei Kindern zutrifft (Statistisches Bundesamt 2018, S. 234). Während unter alleinerziehenden Müttern $28 \%$ und unter alleinerziehenden Vätern $20 \%$ ein monatliches Nettoeinkommen von unter 1300 EUR aufweisen, sind dies bei Ehepaaren nur $2 \%$ (Statistisches Bundesamt 2018, S. 61). Auch die Gesundheit Alleinerziehender ist schlechter als die von Eltern, die in einer Partnerschaft leben. Das im Vergleich zu anderen Lebensformen besonders hohe Armutsrisiko und die stärkere gesundheitliche Gefährdung macht diese Lebensform für die Erforschung sozialer und gesundheitlicher Ungleichheiten interessant. Mit Blick auf diese spezielle Gruppe stellt sich die Frage, wie sich das hohe Armutsrisiko und die gesundheitlichen Risiken erklären lassen.

Vor dem Hintergrund der diesen Band leitenden Fragestellung, welche Rolle die soziologische Netzwerkforschung für die Analyse gesundheitlicher Ungleichheiten spielen kann, soll hier am Beispiel der Studien zur Lebensform 
„,alleinerziehend“ verdeutlicht werden, inwieweit die Netzwerkforschung hier eine Rolle spielt und zur Erklärung gesundheitlicher Ungleichheiten beitragen kann. Zunächst wird dafür der aktuelle Forschungsstand zur Gesundheit von Alleinerziehenden präsentiert. Anschließend wird aufgearbeitet, was über deren soziale Integration, soziale Beziehungen und soziale Netzwerke bekannt ist, und welche Forschungslücken hier ggf. noch bestehen. Im darauffolgenden Abschnitt werden dann die Wechselwirkungen zwischen sozialen Netzwerken und der Gesundheit Alleinerziehender analysiert. Den Abschluss bilden ein Fazit und Forschungsdesiderata.

\section{Die Gesundheit Alleinerziehender}

Die Gesundheit Alleinerziehender wurde in den letzten Jahrzehnten häufiger untersucht. Im Fokus stehen dabei wegen des geringen Anteils alleinerziehender Väter in der Bevölkerung zumeist alleinerziehende Mütter. Nur wenige Studien beziehen alleinerziehende Väter ein oder stellen sie gar in den Mittelpunkt. Das Bild, das diese Studien seit Jahrzehnten für Deutschland wie für andere westliche Staaten zeigen, erscheint eindeutig: Alleinerziehende sind weniger gesund als Mütter und Väter, die in anderen Lebensformen leben. Dies gilt sowohl für die körperliche als auch für die seelische Gesundheit. So schätzen z. B. alleinerziehende Mütter ihre Gesundheit schlechter ein als Mütter, die in einer Partnerschaft leben (Chiu et al. 2016; Helfferich et al. 2003; Rousou et al. 2013; Sperlich et al. 2011; Van de Velde et al. 2014).

Alleinerziehende Mütter geben häufiger körperliche Beschwerden an, aber auch psychische wie Angst und Depressivität (Franz et al. 2003; Helfferich et al. 2003; Sperlich et al. 2011; Van de Velde et al. 2014). Auch weisen sie ein geringeres Wohlbefinden und eine geringere Lebenszufriedenheit auf als Eltern in Paarbeziehungen (Helfferich et al. 2003; Kohler et al. 2005; Osborne et al. 2012; Vignoli et al. 2014; Winkelmann und Winkelmann 1998). Das Gesundheitsverhalten alleinerziehender Mütter gilt als risikoreicher, so ist der Anteil der regelmäßigen Raucherinnen unter den alleinerziehenden Müttern fast doppelt so hoch (46\%) wie unter den verheirateten Müttern (24\%) (Helfferich et al. 2003, S. 16), auch achten alleinerziehende Mütter weniger auf eine gesunde Ernährung und sind etwas weniger sportlich aktiv als verheiratete Mütter (ebd.). Ähnliche Befunde zur subjektiven Gesundheit, zu körperlichen wie psychischen Beschwerden wie auch zum Rauchverhalten finden sich auch für alleinerziehende Väter (Benzeval 1998; Chiu et al. 2016; Cooper et al. 2008; Sperlich et al. 2011; 
Westin und Westerling 2006), wenngleich bei Vätern gelegentlich auch kein Unterschied zu Vätern in Partnerschaft gemessen werden kann (Domanska et al. 2013; Helfferich et al. 2003).

\section{Theoretische Erklärungsansätze für die schlechtere Gesundheit Alleinerziehender}

Wenn wir danach fragen, wie die höhere gesundheitliche Belastung Alleinerziehender erklärt werden kann, finden wir häufig Ansätze, die auf die schlechteren sozioökonomischen Lebensbedingungen von Alleinerziehenden (geringeres Einkommen, schlechtere materielle Ausstattung, Arbeitslosigkeit) verweisen (Benzeval 1998). Dies scheint auch insbesondere für alleinerziehende Väter eine wichtige Rolle zu spielen (Chiu et al. 2016). In vielen dieser Studien wird aber auch deutlich, dass der Blick auf sozioökonomische Faktoren allein nicht ausreicht (Benzeval 1998), oder dass andere Faktoren einen stärkeren Erklärungsgehalt bieten (Cairney et al. 2003). Dazu gehören zum Beispiel 1) Stress während des Prozesses der Trennung/Scheidung/Verwitwung oder das stärkere Erleben von Stress generell (Cairney et al. 2003), 2) gesellschaftliche Stigmatisierung der Einelternschaft (Evans et al. 1994), und 3) Selektionseffekte, d. h., dass erkrankte Personen oder Personen mit geringerem Wohlbefinden eher alleinerziehend werden als gesunde (Gerstel et al. 1985; Riessman und Gerstel 1985).

Verbreitet sind auch Erklärungsansätze, die soziale Beziehungen einbeziehen, z. B. das Fehlen eines Vertrauten, einer intimen Bezugsperson oder einer physisch im Haushalt präsenten unterstützenden Person (Anson 1989; Brown et al. 1993; Brown und Harris 1993) oder auch Kontakte zu und die Unterstützung von anderen Personen allgemein. Auf solche Forschungsarbeiten, die soziale Kontakte und die soziale Unterstützung Alleinerziehender in den Mittelpunkt stellen, wird im folgenden Kapitel näher eingegangen.

\section{Soziale Beziehungen Alleinerziehender}

Soziale Beziehungen und soziale Unterstützung sind schon seit einigen Jahrzehnten wichtige Faktoren im Rahmen der Erforschung der Auswirkungen von Scheidung und Trennung. Studien zu Alleinerziehenden, die ggf. nie verheiratet waren oder nie in einer Partnerschaft lebten, und anders als Geschiedene per Definition immer Kinder im Haushalt haben, sind eher jüngeren Datums. 
Die Scheidungsforschung zeigt v. a., dass eine Trennung einen Verlust sozialer Kontakte und ihrer positiven Auswirkungen mit sich bringt. So verändert der Verlust eines Partners oder einer Partnerin nicht nur die Alltagsorganisation, da ein Mitstreiter oder eine Mitstreiterin in allen anfallenden Aufgaben fehlt, auch geht damit eine wichtige Quelle für emotionale Unterstützung und soziale Wertschätzung verloren (z. B. Gerstel et al. 1985; Myers et al. 1975). Mit der Trennung kommt es auch zum Verlust weiterer Kontakte (Milardo 1987), z. B. weil gemeinsame Freunde Loyalitätskonflikte erleben und die Freundschaft nur zu einem der beiden Partner aufrecht erhalten (Terhell et al. 2004), ebenso ist es für viele Eltern schwierig, nach der Trennung den Kontakt zu der Schwiegerfamilie aufrecht zu erhalten (Dearlove 1999). Häufig gilt daher seit langem soziale Isolation als wesentliche Folge einer Scheidung (Jauch 1977), und damit verbunden auch ein Verlust an sozialer Unterstützung. Die aktuelleren empirischen Befunde $\mathrm{zu}$ sozialer Unterstützung nach einer Trennung sind jedoch nicht konsistent: Einige Studien finden, dass geschiedene Eltern verstärkte Unterstützung durch die eigenen Eltern erhalten und weiterhin Unterstützung durch die Schwiegereltern, während andere einen Rückgang sozialer Unterstützung konstatieren (Harknett und Knab 2007). Relevant ist hierbei auch, wie viel Zeit seit der Trennung vergangen ist. So zeigen neuere Längsschnittstudien ein differenzierteres Bild, was die längerfristigen Folgen einer Trennung angeht: Zwar kommt es zu Kontaktverlusten kurz nach der Scheidung, etwa die Hälfte der Befragten kompensiert diese Verluste jedoch in den folgenden Jahren. Im Zeitverlauf geht die Anzahl der Verwandten zurück (wahrscheinlich durch den Verlust der Schwiegerfamilie), der Kontakt zu Freundinnen und Freunden nimmt zu, für Männer auch die Unterstützung von Freundinnen und Freunden sowie Bekannten (Terhell et al. 2004).

Da eine Scheidung nicht der einzige Weg in die Einelternschaft ist, und geschiedene Elternteile auch nicht notwendigerweise mit ihren Kindern zusammenleben, können die Ergebnisse der Scheidungsforschung nicht unbedingt auf Alleinerziehende übertragen werden. So ist beispielsweise bekannt, dass Frauen nach einer Verwitwung mehr Unterstützung von Verwandten, Freunden und Nachbarn erhalten als nach einer Trennung (Kalmijn 2012) und dass sich die sozialen Netzwerke und der Erhalt von Unterstützung bei geschiedenen und nie verheirateten Alleinerziehenden unterscheiden (Nestmann und Stiehler 1998). Studien, die die sozialen Beziehungen Alleinerziehender differenziert nach ihrem Weg in die Einelternschaft untersuchen, liegen jedoch kaum vor.

Vergleicht man die sozialen Beziehungen von Alleinerziehenden mit denen von Eltern in Paarbeziehungen so zeigt sich in vielen Bereichen ein reduzierter Umfang: Alleinerziehende haben weniger Kontakte zu Verwandten als Eltern in Partnerschaften (Cairney et al. 2003; Cochran et al. 1990), weniger Kontakte 
zur Schwiegerfamilie (Dearlove 1999) und weniger Kontakte zu Freunden (Cairney et al. 2003). Auch sind sie seltener in Vereinen oder Organisationen aktiv als Eltern in Partnerschaften (ebd.). Die Befunde zur sozialen Unterstützung sind uneinheitlich (Lye 1996): So zeigen manche Studien, dass Alleinerziehende weniger Unterstützung als Eltern in Partnerschaften erhalten (Cairney et al. 2003; Reeves et al. 1994; Targosz et al. 2003); andere Studien zeigen, dass v. a. alleinerziehende Mütter nach einer Scheidung mehr Unterstützung erhalten (Hogan et al. 1990; Marks und McLanahan 1993). Alleinerziehende können allerdings in besonderem Maße von sozialer Unterstützung profitieren (Balaji et al. 2007; Harknett 2006), denn v. a. Unterstützung bei der Kinderbetreuung ermöglicht es für viele Alleinerziehende, einer Erwerbstätigkeit nachzugehen, und Familienarbeit und Erwerbstätigkeit zu vereinbaren, und damit im besten Fall einer Armutslage zu entgehen (Ciabattari 2007; Cook 2012).

Während sich die Unterstützungsforschung v. a. mit der Wahrnehmung und dem Erhalt bestimmter Formen sozialer Unterstützung beschäftigt, nimmt die soziale Netzwerkforschung eine erweiterte Perspektive ein, indem sie die Bedeutung sozialer Beziehungen betrachtet und dabei auch die strukturellen Eigenschaften der Beziehungsnetzwerke einbezieht (Smith und Christakis 2008). Die Netzwerke Alleinerziehender sind sehr heterogen in Struktur und Funktion: es gibt große und kleine, dichte und weniger dichte, unterstützende und weniger unterstützende (Campbell et al. 2016; Keim 2018; Lumino et al. 2016; Niepel 1994). Sozial weitgehend isolierte Personen finden sich eher selten, aber besonders in Armutslagen (ebd.). Durch die Trennung verlorene Kontakte werden häufig durch neue oder die Intensivierung bereits bestehender Beziehungen ersetzt (Niepel 1994).

Zur Zusammensetzung der Netzwerke finden sich widersprüchliche Befunde. Einigkeit besteht weitgehend darüber, dass Frauen unter den Personen, die Unterstützung leisten, dominieren (Attree 2005; Keim 2018; Niepel 1994). Während manche Studien eine Dominanz von Verwandten aufzeigen (Lumino et al. 2016), präsentieren andere Studien eine Dominanz von Freundschaften (Niepel 1994). In jedem Fall wird deutlich, dass die Netzwerke häufig sehr heterogen in ihrer Zusammensetzung sind und dass die Variationsbreite unterschiedlicher Netzwerkstrukturen und Kompositionen groß ist. Interessant sind daher Studien, die Netzwerktypologien entwickeln. Davon gibt es bisher allerdings recht wenige, es konnten in den letzten fast 40 Jahren nur drei solcher Studien in Deutschland und den USA gefunden werden. Diese stützen sich zudem auf sehr kleine und sehr unterschiedliche Samples, was sie kaum vergleichbar macht: 45 weiße geschiedene, zumeist erwerbstätige Mütter in den USA (McLanahan et al. 1981), 
20 erwerbstätige und erwerbslose Alleinerziehende in Bielefeld (Niepel 1994), 26 erwerbslose Alleinerziehende in Mecklenburg-Vorpommern (Keim 2018).

McLanahan et al. (1981) identifizieren in ihrer Studie über geschiedene Mütter drei Netzwerktypen: 1) „Rückkehr in die Herkunftsfamilie“, recht klein und dicht, dominiert von Verwandten, 2) ,erweitertes Netzwerk“, recht groß, wenig dicht und heterogen in der Zusammensetzung und 3) „Aufrechterhaltung oder Neugründung einer conjugalen Beziehung mit dem Ex-Partner oder einem neuen Partner", enthält Verwandte und Freunde, kann unterschiedlich groß und dicht sein. Niepel (1994) unterscheidet in ihrem Sample weiblicher Alleinerziehender 1) „Freundschaftsnetzwerke“, relativ kleine und wenig dichte Netzwerke, in denen Freunde dominieren, von 2) „Familien- und Freundschaftsnetzwerken“, große Netzwerke, in denen beide Rollenbeziehungen häufig vorkommen, und 3) „Familiennetzwerke“, kleine und dichte Netzwerke, in denen Verwandtschaftsbeziehungen dominieren. Keim (2018) unterscheidet in ihrer Studie über erwerbslose weibliche Alleinerziehende 1) „Familienorientierte“ und 2) „Conjugale Netzwerke", die vor allem verwandtschaftliche Beziehungen enthalten, sei es zu eigenen Verwandten oder zu Angehörigen des Partners, 3) „Erweiterte Netzwerke", die groß und wenig dicht sind und sich aus unterschiedlichen Beziehungstypen zusammensetzen, und 4) „Eingeschränkte Netzwerke“, die sehr klein sind und einen hohen Anteil an institutionellen Helfern aufweisen. Gemeinsam haben die drei Typologien, dass die Variationsbreite der Netzwerke Alleinerziehender hoch ist, von besonders großen und wenig dichten Netzwerken („Erweitertes Netzwerk“ bei McLanahan und Keim und „Familien- und Freundschaftsnetzwerk" bei Niepel) bis zu kleinen, dichten und verwandtschaftsdominierten Netzwerken. Allein die „Eingeschränkten Netzwerke“ bei Keim (2018) zeugen von einer gewissen sozialen Isolation. In diesen Netzwerken finden sich aber oft institutionelle Helfer, die wichtige Unterstützungsfunktionen übernehmen. Die Entwicklung einer Typologie basierend auf einem größeren Sample steht noch aus.

Fazit: Ob es um soziale Kontakte, soziale Beziehungen, soziale Unterstützung oder soziales Kapital geht, die vorgestellten Studien machen deutlich, dass Alleinerziehende über eine andere soziale Einbettung verfügen als verheiratete Eltern. Konzepte wie soziale Unterstützung werden dabei häufiger untersucht, netzwerkanalytische Studien sind eher selten. Auffällig ist, dass der Begriff „Netzwerke“ hier häufig als Metapher genutzt wird („Unterstützungsnetzwerke“) und für eine Vielzahl sozialer Beziehungen und Unterstützungsformen stehen kann. Seltener wird in diesen Studien, die von „Unterstützungsnetzwerken“ sprechen, auch tatsächlich die Struktur dieser Beziehungen genauer untersucht und netzwerkanalytische Instrumente angewandt. 


\section{$5 \quad$ Welche Rolle spielen soziale Beziehungen für die Gesundheit Alleinerziehender?}

Viele Studien konnten in den letzten Jahrzehnten zeigen, dass soziale Beziehungen bedeutsam für das Wohlbefinden, die Gesundheit und das Gesundheitsverhalten sind. Dies gilt auch für Alleinerziehende. Recht gut erforscht ist das Konzept der sozialen Unterstützung: Alleinerziehende profitieren davon, dass soziale Unterstützung dazu beiträgt, stressreiche Lebenssituationen abzumildern und damit positiv auf das Wohlbefinden wirkt (Campbell et al. 2016; Ciabattari 2007; Harknett 2006). Soziale Unterstützung korreliert auch negativ mit konkreten Krankheitsbildern: So geht eine geringere wahrgenommene soziale Unterstützung mit einem häufigeren Auftreten von depressiven Symptomen (Cairney et al. 2003; Harknett 2006) und anderen psychischen Erkrankungen (Franz et al. 2003) einher. Im Vergleich zu Müttern in Paarbeziehungen sind alleinerziehende Mütter nicht nur vermehrt Stressoren ausgesetzt, sondern verfügen auch über weniger Unterstützung (Cairney et al. 2003) und das Zusammenspiel aus geringerem sozialen Status, höherer Anzahl von Stressoren und geringerer Unterstützung kann die Unterschiede in der Depressionsneigung zwischen Müttern, die mit einem Erwachsenen im Haushalt zusammenleben, und Alleinerziehenden fast vollständig erklären (Targosz et al. 2003).

Ein differenzierteres Bild ergibt sich, wenn man die Zusammenhänge zwischen sozialem Status und sozialer Unterstützung genauer analysiert. Für Personen mit geringerem sozialen Status kann soziale Unterstützung deutlich entlastend wirken, so verringert der Zugang zu sozialer Unterstützung Konflikte in der Vereinbarung von Familie und Beruf für unverheiratete Mütter, v. a. für solche mit niedrigerem sozialen Status (Ciabattari 2007). Soziale Beziehungen generieren aber auch Kosten. Erhaltene Unterstützung muss häufig erwidert werden, was besonders für Personen mit geringerem sozialen Status, wie z. B. für erwerbslose Alleinerziehende, schwierig sein kann. Die Reziprozitätserwartungen üben sozialen Druck aus und belasten dann nicht nur das Wohlbefinden, sondern können längerfristig auch zu Kontaktabbrüchen und dem Verzicht auf soziale Unterstützung führen (Andreotti 2006; Cook 2012). Ob aktiver Verzicht auf Unterstützung oder mangelnder Zugang zu Unterstützungsmöglichkeiten, individuelle Benachteiligung wird durch solche Beziehungseffekte verstärkt: Für Alleinerziehende mit geringer Bildung, in prekärer Beschäftigung und Armutslagen, die Unterstützung am meisten benötigen, ist sie am wenigsten verfügbar (Brown und Moran 1997; Harknett 2006). Dieser Mangel an Unterstützung wiederum steht in enger Beziehung mit psychischen Erkrankungen (Simons et al. 1993). 
Soziologische Netzwerkstudien, die sich mit den Beziehungsstrukturen und ihren Auswirkungen beschäftigen und dabei soziale Wirkmechanismen betrachten, die über soziale Unterstützung hinausgehen (vgl. Berkman und Glass 2000), sind in der Alleinerziehendenforschung noch sehr selten. Dennoch finden sich Studien, die zeigen, dass soziale Netzwerke für die Gesundheit Alleinerziehender oder Geschiedener besonders relevant sind. So zeigt beispielsweise die Studie von Gerstel et al. (1985), dass die Charakteristika sozialer Netzwerke den Zusammenhang zwischen Familienstand (Geschieden oder Verheiratet) und psychischer Gesundheit zu einem hohen Grad erklären kann: für Männer $60 \%$ und für Frauen $57 \%$ der Unterschiede in der psychischen Gesundheit zwischen Verheirateten und Geschiedenen (ebd., S. 95). Dabei finden sich bedeutsame Unterschiede zwischen Männern und Frauen. Während für Frauen neben Netzwerkcharakteristika vor allem materielle Faktoren (v. a. das Einkommen) eine protektive Wirkung haben, ist es bei Männern vor allem die Struktur und Zusammensetzung des Netzwerks. Für beide Geschlechter vermindern Netzwerke, die nicht als Belastung empfunden werden und keine Einsamkeitsgefühle aufkommen lassen, das Risiko einer psychischen Erkrankung (ebd.).

Befunde für die genaue Wirkung spezifischer Netzwerkstrukturmaße auf die Gesundheit Alleinerziehender sind rar und meist uneinheitlich. So sind einerseits bei Geschiedenen große Netzwerke mit Glück und Wohlbefinden assoziiert, unabhängig davon, welche Inhalte in diesen Netzwerken ausgetauscht werden (Gerstel et al. 1985), bei Frauen stehen zudem große Netzwerke mit psychischer Gesundheit in Zusammenhang (ebd.). Andererseits bieten kleinere Netzwerke aus der Sicht von alleinerziehenden Müttern mehr und bessere Unterstützung als größere (Malo 1994), was Gesundheitseffekte haben kann. Wichtig ist festzuhalten, dass es sich bei den genannten Studien um Querschnittuntersuchungen handelt, die keine Aussagen über Ursache und Wirkung treffen können. Denkbar ist daher sowohl, dass große Netzwerke protektiv wirken, als auch, dass gesundheitlich weniger belastete Frauen eher in der Lage sind, größere Netzwerke zu unterhalten.

Hinsichtlich der Netzwerkdichte zeigen erste Befunde, dass gängige theoretische Überlegungen zur Rolle der Netzwerkdichte und der sozialen Unterstützung nicht notwendigerweise auf Alleinerziehende zutreffen. Allgemein wird angenommen, dass dichte Netzwerke eher instrumentelle und emotionale Unterstützung leisten als weniger dichte, diese stehen vielmehr in einem positiven Zusammenhang mit dem Zugang zu Informationen und neuen sozialen Kontakten (Granovetter 1973; Mitchell 1969). Nach einer Studie von McLanahan, Wedemyer und Adelberg von 1981 empfinden Alleinerziehende die Unterstützung durch Verwandte (die meist dichte Netzwerke bilden) als weniger befriedigend 
als die Unterstützung durch Freunde in einem Netzwerk mit geringer Dichte. Ein dichtes Netzwerk mit vielen Verwandten geht bei Alleinerziehenden mit einer geringeren Lebensqualität einher (Leslie und Grady 1985). Auch können weniger dichte Netzwerke flexiblere Unterstützung leisten und daher dabei helfen, besser mit einer neuen Situation zurechtzukommen (Gerstel et al. 1985), auf diesem Weg schützen sie vor allem Männer vor psychischen Erkrankungen nach einer Scheidung (ebd.).

Die Netzwerkforschung öffnet nicht nur den Blick für komplexe Beziehungsgeflechte, sondern auch für negative oder ambivalente Beziehungsinhalte. So können soziale Beziehungen nicht nur unterstützend, sondern auch konflikthaft, gelegentlich auch beides gleichzeitig sein. Im Kontext von Trennungs- und Scheidungsprozessen sind negative oder ambivalente Beziehungen besonders relevant, da konfliktbehaftete Beziehungen zu den ehemaligen Partnerinnen und Partnern aber auch deren Eltern, Verwandten oder Freunden nicht ohne Weiteres gekappt werden können, da sie oft wichtige Bezugspersonen für die eigenen Kinder sind. Studien über Alleinerziehende, die negative Beziehungsinhalte einbeziehen, sind rar. Die Studie von Gerstel et al. (1985) fragt neben einzelnen Unterstützungsdimensionen auch danach, ob soziale Beziehungen als Belastung empfunden werden. Die Analyse zeigt, dass Netzwerke, die nicht als Belastung empfunden werden, mit einem geringeren Risiko einer psychischen Erkrankung im Zusammenhang stehen (Gerstel et al. 1985). Auch die Studie von Samuelsson (1994) zeigt, dass Konflikte und negative Kontakte die psychische Gesundheit belasten. Empirisch wissen wir nicht nur bei Alleinerziehenden wenig über das Zusammenspiel von Unterstützung und Konflikten in ihren gesundheitlichen Folgen. Neuere Forschung zeigt, dass bei Studierenden, die ein hohes Maß an Unterstützung erhalten, soziale Konflikte nicht mit ihrem Wohlbefinden korrelieren, während für Personen, die wenig Unterstützung erhalten, soziale Konflikte das Wohlbefinden negativ beeinflussen (Abbey et al. 2010). Hier besteht weiterer Forschungsbedarf.

Eine besonders differenzierte Analyse von Netzwerkstrukturen und ihrer Wirkung auf das individuelle Wohlbefinden von geschiedenen Müttern legten McLanahan, Wedemeyer und Adelberg (1981) vor. Sie haben eine Netzwerktypologie gebildet, in der jeder der vier Netzwerktypen auf seine spezifische Weise mit der Gesundheit Alleinerziehender im Zusammenhang steht. Wesentlich dafür, ob die Netzwerkstruktur als gesundheitsförderlich oder -hinderlich erlebt wird, ist dabei nicht die Struktur selbst, sondern ob sie zur Rollenorientierung der Geschiedenen passt. Geschiedene Mütter, die die sozialen Rollen, die sie vor der Scheidung 
hatten, beibehalten möchten, fühlen sich am besten durch dichte Netzwerke unterstützt, während Mütter, die mit der Scheidung auch ihre sozialen Rollen verändern möchten, sich am besten von weniger dichten Netzwerken unterstützt fühlen. Sind diese Frauen in gänzlich anders strukturierte Netzwerke eingebettet, so geht das mit einem geringeren Wohlbefinden einher. Die Autoren plädieren deshalb dafür, dass spezifische Netzwerkstrukturen und Unterstützungsmöglichkeiten nicht per se für alle die gleiche gesundheitsförderliche oder -hinderliche Wirkung entfalten, sondern dass es auf die Unterstützungsbedarfe ankommt.

Keim (2018) zeigt hingegen, dass es unter erwerbslosen Alleinerziehenden zwei Netzwerkstrukturtypen gibt, die generell mit besonders hohem, respektive geringem Wohlbefinden einhergehen. Dabei handelt es sich zum einen um große und wenig dichte ,Erweiterte Netzwerke“, die eine hohe Heterogenität aufweisen. Personen, die in diese Art von Netzwerk eingebunden sind, erhalten vielfältige soziale Unterstützungsleistungen und sind auch selbst in der Lage, ihre Netzwerkpartner zu unterstützen und damit reziproke Beziehungen zu pflegen. Das Wohlbefinden von Personen, die diesen Netzwerktyp eingebunden sind, ist sehr hoch. Auch hier finden sich Wechselwirkungen zwischen Wohlbefinden und Netzwerkstruktur. Die als hilfreich bewertete Unterstützung fördert das Wohlbefinden, aber das hohe Wohlbefinden und der geringe Stresslevel tragen auch dazu bei, reziproke Unterstützungsbeziehungen zu pflegen, Konflikte konstruktiv anzugehen und den Aufbau neuer Beziehungen zu wagen. In starkem Kontrast dazu stehen zum anderen ,Eingeschränkte Netzwerke“, kleine Netzwerke mit einem hohen Anteil an institutionellen Helfern. Befragte, die in diese Netzwerke eingebettet sind, können oft nur durch den Einsatz der institutionellen Helfer als hilfreich bewertete Unterstützung erhalten, die Unterstützung kann die empfundenen Belastungen aber nur teilweise und kurzfristig abpuffern, das Wohlbefinden ist im Vergleich zu den anderen befragten Alleinerziehenden stark eingeschränkt. Eine aktive Netzwerkpflege, zum Beispiel auch durch das Leisten von Unterstützung, oder der Aufbau neuer Kontakte ist kaum möglich. Zwischen diesen beiden Polen liegen zwei Netzwerktypen, in denen verwandtschaftliche Kontakte zu der Herkunftsfamilie oder der Familie des alten/neuen Partners dominieren. Hier treten häufiger Belastungslagen auf als bei Personen mit erweiterten Netzwerken, diese können aber meist recht gut durch die Unterstützung in den Netzwerken abgepuffert werden, es bleibt genug Zeit und Kraft für die Pflege der bestehenden, und den Aufbau neuer Beziehungen. 


\section{$6 \quad$ Fazit und Forschungsdesiderate}

Mittlerweile gibt es einige Studien, die die Rolle der sozialen Einbettung Alleinerziehender für ihre Gesundheit und ihr Wohlbefinden in den Blick nehmen und die zeigen können, dass spezifische Netzwerkstrukturen einen bedeutsamen Einfluss haben. Es besteht aber noch weiterer Forschungsbedarf, der im Folgenden zusammengestellt werden soll:

1. Die Wege in die Einelternschaft sind vielfältig, Befunde aus der Scheidungsforschung sowohl zu Gesundheit wie auch zu sozialen Beziehungen lassen sich daher nicht unbedingt auch auf Alleinerziehende übertragen, hier sind weitere Studien spezifisch zur Lebensform "Alleinerziehend“ notwendig. Dabei sollte auch das Sorge- und Umgangsrecht berücksichtigt werden, so bieten Wechselmodelle (das Kind lebt zeitweise jeweils bei Mutter und Vater) sicherlich andere Vorteile und Herausforderungen als das alleinige Sorge- und Umgangsrecht, und sind mit anderen Formen der sozialen Einbindung verknüpft.

2. Studien, die die Rolle soziale Beziehungen für die Gesundheit Alleinerziehender in den Mittelpunkt stellen, beschäftigen sich zumeist mit Konzepten wie sozialer Unterstützung, strukturelle Netzwerkanalysen sind selten. Hier besteht noch Forschungsbedarf. Zu fragen ist:

a. Wie verändern sich die Netzwerkstrukturen mit dem Übergang in ein Leben als Alleinerziehende?

b. Welche Rolle spielen spezifische Netzwerkstrukturen für die Gesundheit? Gibt es neben einzelnen be- und entlastenden Beziehungen auch Strukturen des gesamten Netzwerks, die eine besonders be- oder entlastende Wirkung haben?

c. Welche Wirkmechanismen neben sozialer Unterstützung sind relevant?

3. Die Rolle sozialer Netzwerke für die Reproduktion oder Kompensation sozialer und gesundheitlicher Ungleichheiten ist für Alleinerziehende noch kaum erforscht. Die empirische Forschung weist in zwei Richtungen: Zum einen gehen ökonomische Mangellagen häufig mit einem kleineren Netzwerk einher, das in geringerem Maße Unterstützung bereitstellen kann. Da sich sowohl ein Mangel an ökonomischen als auch an sozialen Ressourcen negativ auf die Gesundheit auswirken kann, eine schlechte Gesundheit wiederum die Ausstattung mit Ressourcen negative beeinflusst, deutet sich eine Abwärtsspirale an, in der sich Ressourcenausstattung und Gesundheit stetig weiter verschlechtern. Zum anderen zeigen empirische Studien aber auch, dass sowohl 
Kontaktverluste durch eine Trennung kompensiert werden können als auch, dass soziale Ressourcen einen Mangel an ökonomischen Ressourcen kompensieren können. Damit könnten soziale Netzwerke von Alleinerziehenden eine gesundheitsförderliche Wirkung haben, indem sie negative Gesundheitseffekte von Trennung, Armut und sozialer Ungleichheit abpuffern. Unklar ist jedoch, inwieweit und unter welchen Bedingungen sie das tun oder sich eine negative Spirale entwickelt.

a. Besonders wünschenswert wären hier Längsschnittstudien, die kurz- und längerfristige Veränderungen in der Lebensform, im sozialen Status, in sozialen Netzwerken und der Gesundheit in ihren Bedingungen und Konsequenzen analysieren.

b. Sinnvoll sind aber auch Querschnittserhebungen z. B. unter Alleinerziehenden mit unterschiedlichem sozialen Status oder unterschiedlicher Verweildauer in der Lebensform, die zeigen können, inwieweit sich diese Gruppen in ihrer Netzwerkstruktur und Gesundheit unterscheiden.

c. Qualitative Studien können die Bedeutung sozialer Beziehungen und spezifischer Netzwerkstrukturen für die Alleinerziehenden näher beleuchten und aufzeigen, auf welche Weise, durch welche Mechanismen, unter welchen Bedingungen und in welchen Kontexten soziale Netzwerke zu einer Reproduktion sozialer Ungleichheiten beitragen oder soziale Ungleichheiten kompensieren können. Auch fehlen vertiefende qualitative Studien, die deutlich machen können, wie Personen aus unterschiedlichen sozialen Schichten mit einer Trennung umgehen, und was dies für ihre sozialen Beziehungen bedeutet. Diese können auch in den Mittelpunkt rücken, wie Alleinerziehende ihre sozialen Kontakte bewerten. So muss ein kleineres Netzwerk nicht unbedingt mit einer geringeren sozialen Unterstützung einhergehen, vielleicht wird es auch als positiv erlebt, da Kontakte wegfallen, für deren Pflege vorher Zeit und Mühe investiert werden musste.

d. Häufig werden soziale Beziehungen nur in ihren vorteilhaften Auswirkungen untersucht, sie können jedoch Konflikte beinhalten und Stress auslösen (Gräbe 1991). Gerade für Alleinerziehende mit niedrigerem sozialen Status kann die Annahme von Unterstützung belastend sein (Cook 2012). Diese negativen Aspekte von sozialen Beziehungen werden noch viel zu selten in Netzwerkstudien einbezogen, daher ist zu fragen: Wie lässt sich das komplexe Zusammenspiel von Unterstützung und Konflikten in sozialen Netzwerken fassen, und welche Rolle spielen hierfür konkrete Netzwerkstrukturen? 
4. Unterstützungsnetzwerke sind nicht nur privater Natur, auch von institutioneller Seite wird Unterstützung für Alleinerziehende bereitgestellt, die nicht nur in das persönliche Netzwerk Alleinerziehender integriert werden kann (Familienhelfer etc.), sondern auch selbst netzwerkförmig organisiert sein kann (siehe Bundesministerium für Familie, Senioren, Frauen und Jugend 2013). Auch bezüglich des Zusammenwirkens privater und institutionalisierter Unterstützung besteht Forschungsbedarf. So wirft sich hier die Frage auf, ob institutionalisierte Unterstützung private Unterstützung verdrängt und damit das soziale Gefüge und die Verfügbarkeit von Ressourcen weiter schwächt, oder ob sie nicht gerade besonders in schweren Belastungslagen Unterstützung überhaupt erst ermöglicht, da einzelne Unterstützter nun nicht mehr durch die notwendige Unterstützung überlastet werden.

\section{Leseempfehlungen}

Gerstel, N., Kohler Riessman, C., \& Rosenfield, S. (1985): Explaining the Symptomatology of Separated and Divorced Women and Men: The Role of Material Conditions and Social Networks. Social Forces 64 (1), S. 84-101. Diese US-amerikanische Studie aus den 1980er Jahren interessiert sich dafür, wodurch Ehen gesundheitsprotektiv wirken. Die Autorinnen analysieren Daten der Northern California Community Study zur Gesundheit von Verheirateten und Geschiedenen/getrennt Lebenden. Dabei handelt es sich um eine frühe Studie mit netzwerkanalytischer Perspektive, die auch gezielt Netzwerkstrukturen untersucht. Sie zeigt die Erklärungskraft sozialer Netzwerke für Unterschiede zwischen Verheirateten und Geschiedenen und eine gesundheitsprotektive Wirkung von Netzwerken geringerer Dichte.

Harknett, Kristen (2006): The Relationship Between Private Safety Nets and Economic Outcomes Among Single Mothers. Journal of Marriage and Family, 68 (1), S. 172-191. Die Autorin zeigt anhand US-amerikanischer Daten, dass Alleinerziehende mit geringer Bildung, in prekärer Beschäftigung und Armutslagen zwar am meisten Unterstützung benötigen, diese aber am wenigsten verfügbar haben. Damit verstärken ihres Erachtens Benachteiligungen auf der Meso-Ebene der sozialen Beziehungen Benachteiligungen auf der individuellen Ebene.

Mc Lanahan, Wedemeyer, Adelberg (1981), Niepel (1994) und Keim (2018), siehe Literaturverzeichnis. Dabei handelt es sich um drei 
methodenintegrative netzwerkanalytische Studien (aus den USA und Deutschland), die sehr differenziert die Strukturen und Funktionen sozialer Netzwerke von Alleinerziehenden/Geschiedenen analysieren und deren Auswirkungen auf Wohlbefinden und Gesundheit untersuchen. Dies mündet jeweils in der Entwicklung einer Typologie sozialer Netzwerke.

\section{Literatur}

Abbey, A., Abramis, D. J., \& Caplan, R. D. (2010). Effects of different sources of social support and social conflict on emotional well-being. Basic and Applied Social Psycho$\log y, 6(2), 111-129$.

Andreotti, A. (2006). Coping strategies in a wealthy city of northern Italy. International Journal of Urban and Regional Research, 30(2), 328-345.

Anson, O. (1989). Marital status and women's health revisited: The importance of a proximate adult. Journal of Marriage and Family, 51(1), 185-193.

Attree, P. (2005). Parenting support in the context of poverty: A meta-synthesis of the qualitative evidence. Health and Social Care in the Community, 13(4), 330-337.

Balaji, A. B., Claussen, A. H., Smith, D. C., Visser, S. N., Morales, M. J., \& Perou, R. (2007). Social support networks and maternal mental health and well-being. Journal of women's health, 16(10), 1386-1396.

Benzeval, M. (1998). The self-reported health status of lone parents. Social Science and Medicine, 46(10), 1337-1353.

Berkman, L. F., \& Glass, T. (2000). Social integration, social networks, social support, and health. In L. F. Berkman \& I. Kawachi (Hrsg.), Social epidemiology (S. 137-173). New York: Oxford University Press.

Brown, G. W., \& Harris, T. O. (1993). Aetiology of anxiety and depressive disorders in an inner-city population: Early adversity. Psychological Medicine, 23(1), 143-154.

Brown, G. W., Harris, T. O., \& Eales, M. J. (1993). Aetiology of anxiety and depressive disorders in an inner-city population: Comorbidity and adversity. Psychological Medicine, 23(1), 155-165.

Brown, G. W., \& Moran, P. M. (1997). Single mothers, poverty and depression. Psychological Medicine, 27(1), 21-33.

Bundesministerium für Familie, Senioren, Frauen und Jugend. (2013). Handbuch Unterstützungsnetzwerke Alleinerziehende, Berlin. https://www.bmfsfj.de/bmfsfj/service/publikationen/handbuch-unterstuetzungsnetzwerke-alleinerziehende/111890. Zugegriffen: 18. Jan. 2019.

Cairney, J., Boyle, M., Offord, D. R., \& Racine, Y. (2003). Stress, social support and depression in single and married mothers. Social Psychiatry and Psychiatric Epidemiology, 38(8), 442-449. 
Campbell, M., Thomson, H., Fenton, C., \& Gibson, M. (2016). Lone parents, health, wellbeing and welfare to work: A systematic review of qualitative studies. BMC Public Health, 16, 188-195.

Chiu, M., Rahman, F., Kurdyak, P., Cairney, J., Jembere, N., \& Vigod, S. (2016). Self-rated health and mental health of lone fathers compared with lone mothers and partnered fathers: A population-based cross-sectional study. Journal of Epidemiology and Community Health, 71(5), 417-423.

Ciabattari, T. (2007). Single mothers, social capital, and work-family conflict. Journal of Family Issues, 28(1), 34-60.

Cochran, M., Larner, M., Riley, D., Gunnarsson, L., \& Henderson, C. R. (1990). Extending families: The social networks of parents and their children. Cambridge: Cambridge University Press.

Cook, K. E. (2012). Social support in single parents' transition from welfare to work: Analysis of qualitative findings. International Journal of Social Welfare, 21(4), 338-350.

Cooper, C., Bebbington, P. E., Meltzer, H., Bhugra, D., Brugha, T., Jenkins, R., Farrell, M., \& King, M. (2008). Depression and common mental disorders in lone parents: Results of the 2000 National Psychiatric Morbidity Survey. Psychological Medicine, 38(3), 335-342.

Dearlove, J. (1999). Lone or alone? A qualitative study of lone mothers on low income with reference to support in their everyday lives. Thesis, University of Warwick. http:// wrap.warwick.ac.uk/36376/1/WRAP_THESIS_Dearlove_1999.pdf. Zugegriffen: 16. Jan. 2015.

Domanska, O., Rattay, P., \& von der Lippe, E. (2013). Sind Alleinerziehende öfter von Rückenschmerzen betroffen als in Partnerschaft lebende Mütter und Väter? Ergebnisse der GEDA-Studie 2009/10. Gesundheitswesen, 75(8/9), A189.

Evans, R. G., Hodge, M., \& Pless, I. B. (1994). If not genetics, then what? Biological pathways and population health. In R. G. Evans, M. L. Barer, \& T. R. Marmor (Hrsg.), Why are some people healthy and others not? The determinants of health populations (S. 161-188). New York: Gruyter.

Franz, M., Lensche, H., \& Schmitz, N. (2003). Psychological distress and socioeconomic status in single mothers and their children in a German city. Social Psychiatry and Psychiatric Epidemiology, 38(2), 59-68.

Gerstel, N., Kohler Riessman, C., \& Rosenfield, S. (1985). Explaining the symptomatology of separated and divorced women and men: The role of material conditions and social networks. Social Forces, 64(1), 84-101.

Gräbe, S. (1991). Reziprozität und Stress in ,Support'-Netzwerken. Neue Perspektiven in der familiensoziologischen Netzwerkforschung. Kölner Zeitschrift für Soziologie und Sozialpsychologie, 43(2), 344-356.

Granovetter, M. (1973). The strength of weak ties. American Journal of Sociology, 78(6), $1360-1380$.

Harknett, K. (2006). The relationship between private safety nets and economic outcomes among single mothers. Journal of Marriage and Family, 68(1), 172-191.

Harknett, K., \& Knab, J. (2007). More kin, less support: Multipartnered fertility and perceived support among mothers. Journal of Marriage and Family, 69(1), 237-253.

Helfferich, C., Hendel-Kramer, A., \& Klindworth, H. (2003). Gesundheit alleinerziehender Mütter und Väter. Gesundheitsberichterstattung des Bundes, 14. http://www.gbe-bund. de/pdf/Heft14.pdf. Zugegriffen: 13. Apr. 2018. 
Hogan, D. P., Hao, L.-X., \& Parish, W. L. (1990). Race, kin networks, and assistance to mother-headed families. Social Forces, 68(3), 797-812.

Jauch, C. (1977). The one-parent family. Journal of Clinical Child Psychology, 6(2), 30-32.

Kalmijn, M. (2012). Longitudinal analyses of the effects of age, marriage, and parenthood on social contacts and support. Advances in Life Course Research, 17(4), 177-190.

Keim, S. (2018). Are lone mothers also lonely mothers? Social networks of unemployed lone mothers in eastern Germany. In L. Bernardi \& D. Mortelmans (Hrsg.), Lone parenthood in the life course (S. 111-140). Cham: Springer Open.

Kohler, H.-P., Behrman, J. R., \& Skytthe, A. (2005). Partner + children=happiness? The effects of partnerships and fertility on well-being. Population and Development Review, 31(3), 407-445.

Leslie, L. A., \& Grady, K. (1985). Changes in mothers' social networks and social support following divorce. Journal of Marriage and Family, 47(3), 663-673.

Lumino, R., Ragozini, G., \& Vitale, M. P. (2016). Investigating social support patterns of single mothers from a social network perspective. International Review of Social Research, 6(4), 182-194.

Lye, D. N. (1996). Adult child-parent relationships. Annual Review of Sociology, 22(1), 79-102.

Malo, C. (1994). Ex-partner, family, friends, and other relationships: Their role within the social network of long-term single mothers. Journal of Applied Social Psychology, 24(1), 60-81.

Marks, N. F., \& McLanahan, S. S. (1993). Gender, family structure, and social support among parents. Journal of Marriage and Family, 55(2), 481-493.

McLanahan, S. S., Wedemeyer, N. V., \& Adelberg, T. (1981). Network structure, social support, and psychological well-being in the single-parent family. Journal of Marriage and the Family, 43(3), 601-618.

Milardo, R. M. (1987). Changes in social networks of women and men following divorce. A review. Journal of Family Issues, 8(1), 78-96.

Mitchell, C. J. (1969). The concept and use of social networks. In C. J. Mitchell (Hrsg.), Social networks in urban situations. Manchester: Manchester University Press.

Myers, J. K., Lindenthal, J. J., \& Pepper, M. P. (1975). Life events, social integration and psychiatric symptomatology. Journal of Health and Social Behavior, 16(4), 421-427.

Nestmann, F., \& Stiehler, S. (1998). Wie allein sind Alleinerziehende? Soziale Beziehungen alleinerziehender Frauen und Männer in Ost und West. Opladen: Leske \& Budrich.

Niepel, G. (1994). Soziale Netze und soziale Unterstützung alleinerziehender Frauen. Opladen: Leske \& Budrich.

Osborne, C., Berger, L. M., \& Magnuson, K. (2012). Family structure transitions and changes in maternal resources and well-being. Demography, 49(1), 23-47.

Reeves, J., Kendrick, D., Denman, S., \& Roberts, H. (1994). Lone mothers: Their health and lifestyle. Health Education Journal, 53, 291-299.

Riessman, C. K., \& Gerstel, N. (1985). Marital dissolution and health: Do males or females have greater risk? Social Science and Medicine, 20(6), 627-635.

Rousou, E., Kouta, C., Middleton, N., \& Karanikola, M. (2013). Single mothers' self-assessment of health. A systematic exploration of the literature. International Nursing Review, 60(4), 425-434. 
Samuelsson, M. A. K. (1994). Associations between the mental health and social networks of children and parents in single-parent families. Acta Psychiatrica Scandinavica, 90(6), 438-445.

Simons, R. L., Beaman, J., Conger, R. D., \& Chao, W. (1993). Stress, support, and antisocial behavior trait as determinants of emotional well-being and parenting practices among single mothers. Journal of Marriage and Family, 55(2), 385-394.

Smith, K. P., \& Christakis, N. A. (2008). Social networks and health. Annual Review of Sociology, 34(1), 405-429.

Sperlich, S., Arnhold-Kerri, S., \& Geyer, S. (2011). Soziale Lebenssituation und Gesundheit von Müttern in Deutschland. Ergebnisse einer Bevölkerungsstudie. Bundesgesundheitsblatt, Gesundheitsforschung, Gesundheitsschutz, 54(6), 735-744.

Statistisches Bundesamt. (2018). Datenreport 2018. Ein Sozialbericht für die Bundesrepublik Deutschland. Bonn.

Targosz, S., Bebbington, P., Lewis, G., Brugha, T., Jenkins, R., Farrell, M., \& Meltzer, H. (2003). Lone mothers, social exclusion and depression. Psychological Medicine, 33(4), $715-722$.

Terhell, E. L., Broese Van Groenou, M. I., \& van Tilburg, T. G. (2004). Network dynamics in the long-term period after divorce. Journal of Social and Personal Relationships, 21(6), 719-738.

Van de Velde, S., Bambra, C., Van der Bracht, K., Eikemo, T. A., \& Bracke, P. (2014). Keeping it in the family: The self-rated health of lone mothers in different European welfare regimes. Sociology of Health \& Illness, 36(8), 1220-1242.

Vignoli, D., Pirani, E., \& Salvini, S. (2014). Family constellations and life satisfaction in Europe. Social Indicators Research, 117(3), 967-986.

Westin, M., \& Westerling, R. (2006). Health and healthcare utilization among single mothers and single fathers in Sweden. Scandinavian Journal of Public Health, 34(2), 182-189.

Winkelmann, L., \& Winkelmann, R. (1998). Why are the unemployed so unhappy? Evidence from panel data. Economica, 65, 1-15.

Open Access Dieses Kapitel wird unter der Creative Commons Namensnennung 4.0 International Lizenz (http://creativecommons.org/licenses/by/4.0/deed.de) veröffentlicht, welche die Nutzung, Vervielfältigung, Bearbeitung, Verbreitung und Wiedergabe in jeglichem Medium und Format erlaubt, sofern Sie den/die ursprünglichen Autor(en) und die Quelle ordnungsgemäß nennen, einen Link zur Creative Commons Lizenz beifügen und angeben, ob Änderungen vorgenommen wurden.

Die in diesem Kapitel enthaltenen Bilder und sonstiges Drittmaterial unterliegen ebenfalls der genannten Creative Commons Lizenz, sofern sich aus der Abbildungslegende nichts anderes ergibt. Sofern das betreffende Material nicht unter der genannten Creative Commons Lizenz steht und die betreffende Handlung nicht nach gesetzlichen Vorschriften erlaubt ist, ist für die oben aufgeführten Weiterverwendungen des Materials die Einwilligung des jeweiligen Rechteinhabers einzuholen.

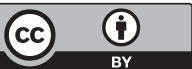

